\title{
Laceration in rat gastrocnemius. Following-up muscle repairing by ultrasound biomicroscopy (in vivo), contractility test (ex vivo) and histopathology ${ }^{1}$
}

\author{
Carlos Martins Ferreira Filho', Ananssa Maíra dos Santos Silva ${ }^{\mathrm{II}}$, Roberto Takashi Sudo ${ }^{\mathrm{III}}$, Christina Maeda Takiya ${ }^{\mathrm{IV}}$, João \\ Carlos Machadov
}

DOI: http://dx.doi.org/10.1590/S0102-86502015001000003

IFellow Master degree, Postgraduate Program in Surgical Sciences, Department of Surgery, School of Medicine, Federal University of Rio de Janeiro (UFRJ), Brazil. Acquisition and interpretation of data, technical procedures, manuscript preparation.

IIFellow Master degree, Postgraduate Program in Drug Development, Department of Basic and Clinical Pharmacology, Biomedical Science Institute, UFRJ, Rio de Janeiro-RJ, Brazil. Technical procedures related to contractility tests, acquisition of data.

IIIPhD, Full Professor, Department of Basic and Clinical Pharmacology, Biomedical Science Institute, UFRJ, Rio de Janeiro-RJ, Brazil. Technical procedures related to contractility tests acquisition of data, design of the contractility study, critical revision.

${ }^{\mathrm{IV}} \mathrm{PhD}$, Associate Professor, Immunopathology Laboratory, Carlos Chagas Filho Institute, UFRJ, Rio de Janeiro-RJ, Brazil. Design of the histopathological study; acquisition, interpretation and analysis of data; critical revision.

${ }^{v}$ Full Professor, Postgraduate Program in Surgical Sciences, Department of Surgery, School of Medicine and Biomedical Engineering Program, COPPE, UFRJ, Rio de Janeiro-RJ, Brazil. Design of the study, acquisition and analysis of data, manuscript writing, English version, supervised all phases of the study.

\section{ABSTRACT}

PURPOSE: Implement a laceration protocol of the rat lateral gastrocnemius (LG) and following-up its repair with ultrasound biomicroscopy (UBM), contractility tests and histology.

METHODS: Sixty-three male Wistar rats were distributed into two groups. One, with sub-groups GI, GII and GIII ( $\mathrm{n}=12$ ), each containing right $\mathrm{LG}$ lacerated $(\mathrm{n}=6)$, control and sham $(\mathrm{n}=3)$ animals. LG muscles in GI, GII and GIII were inspected by UBM (40 $\mathrm{MHz}$ ) immediately after, 14 and 28 days post-surgery and thereafter excised with four (GI), 14 (GII) and 28 (GIII) days post-surgery for histology. Animals in second group were distributed into right LG lacerated and control sub-groups. LG muscles in lacerated sub-group were submitted to contractility tests at four $(n=8), 14(n=8)$ and $28(n=8)$ days post-surgery, while in the control sub-group ( $n=5)$ were submitted to contractility tests along the course of the experiments.

RESULTS: Descriptive findings agreed between the lesion model, muscle repair, UBM images and histology. Contractility results for right LG were different $(\mathrm{p}<0.05)$ between control and injured muscle with four and 14 days post-surgery, at tetanic stimulating frequencies $(50$ and $70 \mathrm{~Hz})$.

CONCLUSION: A laceration protocol of the lateral gastrocnemius was implemented and ultrasound biomicroscopy, contractility and histology findings agreed regarding the following-up of injured muscle repair.

Key words: Muscle, Skeletal. Lacerations. Regeneration. Models, Animal. Microscopy, Acoustic. Rats. 


\section{Introduction}

Eating, drinking, breathing, walking, working and several sport practices are among the several activities of about 640 skeletal muscles that form the human body. Muscle contraction generates force and daily motion of our body, which are fundamental activities for survival and life quality. Plasticity and repair (usually named regeneration) are some of important muscle tissue characteristics that respond to environmental stimulus such as physical exercises and lesions ${ }^{1}$.

Skeletal muscle lesions are common for sympathizers, professionals or not, of any sport modality and in $90 \%$ of the occurrences they are contusion or strain ${ }^{2}$. In general, the incidence of muscle lesions in sports ranges from 10 to $55 \%{ }^{3}$. In addition, several other events that cause skeletal muscle trauma include motorized vehicle accidents, falls, gunfire bullets, explosion, just to mention some.

The recovery of injured muscle tissue follows a timeline that lasts for three to four weeks and the muscle repair consists of molecular and cellular events ${ }^{4}$ responsible for the complete functional recovery through inter-related phases such as degeneration/inflammation, repair and remodeling. Frequently, fibrosis is one of the scar end product that causes functional incapacity, pain and possible lesion recurrence ${ }^{5}$.

The muscular volumetric loss, the fibrosis, the local pain, the tension recurrence, the atrophy and other sequelae of muscle lesions justify scientific investigations to provide relevant and permanent solutions to the issue of muscle repair. In this regard, several scientific investigations confirm possible and potential therapeutic targets related to medicine administered to muscle repair ${ }^{6}$, including cellular therapy, tissue engineering and biotechnology ${ }^{7}$. Even so, the majority of these investigations require methodological approaches able to simulate the human muscle lesions that are commonly observed in the surgicalclinical field, given that the ethical issues are preserved. In this regard, it is important the development of novel models based on experimental laboratory animals, to assist in a clear understanding of the mechanisms equally involved in muscle repair, fibrosis onset and contractility. Under this circumstance, the mouse and rat gastrocnemius muscles have been largely used in investigations related to skeletal muscle related studies ${ }^{8-11}$.

The scientific literature contemplates several experimental studies regarding muscle lesions such as strain, contusion, crushing and laceration. Even so, the model for muscle lesion based on laceration differs from the other types of lesion, because the length of the damage caused to muscle tissue structures, including the membrane, myofibrils and nerves, can lead to functional incapacity through the fibrosis generation ${ }^{12}$.

The present work objective was to implement a controlled and reproducible protocol for acute muscular lesion involving a laceration of the rat lateral gastrocnemius. In addition the work includes the following-up of the muscle repairing process through high resolution ultrasound images, acquired in vivo and employing an image instrumentation named ultrasound biomicroscopy (UBM), adequately scaled to image small animals (mouse and rat), combined with the histological findings of injured muscles and also with the analysis of muscle contractility, ex vivo, electrically stimulated.

\section{Methods}

This investigation was approved by the Ethics Committee for the Use of Animals in Scientific Experiment (protocol 077/14), from the Federal University of Rio de Janeiro, and performed in compliance with the 2013 Brazilian Guide for the Care and Use of Laboratory Animals. The study followed the principles complied with Federal Law No. 11.794/2008, and Decree No. 6,689/2009 that regulated the Law 11.794.

Sixty three male Wistar rats, Rattus norvegicus (Berkenhout, 1769), with body weight between 210 and 300g (7-11 weeks) were distributed into groups $\mathrm{G}_{\mathrm{bmu}}(\mathrm{n}=36)$ and $\mathrm{G}_{\mathrm{cmi}}(\mathrm{n}=29)$, consisting of three and four sub-groups, respectively (Table 1).

The sub-groups in $\mathrm{G}_{\mathrm{bmu}}$ were named GI, GII e GIII, all of them with $n=12$. On each sub-group, six animals (laceration, denoted la1-la6) were submitted to partial laceration of the right LG (lateral gastrocnemius), three (sham, sh1-sh3) were submitted to a skin incision followed by a dissected right LG and other three animals (control, ct1-ct3) did not receive any surgical procedure. Animals in GI had the UBM images, from both LG, acquired immediately after the muscle partial laceration, while the others were inspected by UBM on 14 (GII) and 28 (GIII) days following the surgery. In addition, the animals in GI, GII and GIII were induced to death at four days (GI) and immediately (GII and GIII) after UBM image acquisition. Thereafter, their left and right gastrocnemius muscles were excised for histological analysis.

The sub-groups in $\mathrm{G}_{\mathrm{cmi}}$ were $\mathrm{Gct}(\mathrm{n}=5), \mathrm{G} 04 \mathrm{~d}(\mathrm{n}=8), \mathrm{G} 14 \mathrm{~d}$ $(\mathrm{n}=8)$ and $\mathrm{G} 28 \mathrm{~d}(\mathrm{n}=8)$. Get formed the control group, without any lesion of the LG, while G04d, G14d and G28d formed the groups with partial laceration of the right LG. All animals had their gastrocnemius muscles excised and submitted to the contractility test immediately after death induction at 4 (G04d), 14 G14d e 28(G28d) days following surgery and along the experiments (Gct). 
TABLE 1 - Allocation of the animals on each group and sub-group used in the experiments.

\begin{tabular}{|c|c|c|c|c|c|c|c|c|c|c|c|c|}
\hline \multicolumn{9}{|c|}{$\underset{\mathrm{G}_{\mathrm{bmu}}}{\text { (muscle examined by UBM) }}$} & \multicolumn{4}{|c|}{$\begin{array}{c}\mathrm{G}_{\mathrm{cmi}} \\
\text { (muscle under contractility test) }\end{array}$} \\
\hline & $\begin{array}{c}\text { GI } \\
\text { st-les } \\
0^{+} \text {day }\end{array}$ & & & $\begin{array}{l}\text { GII } \\
\text { st-les } \\
4 \text { day }\end{array}$ & & & $\begin{array}{l}\text { GIII } \\
\text { st-les } \\
8 \text { day }\end{array}$ & & $\begin{array}{l}\text { Gct } \\
\text { no lesion }\end{array}$ & $\begin{array}{c}\text { G04d } \\
\text { post-lesion } \\
04 \text { days }\end{array}$ & $\begin{array}{c}\text { G14d } \\
\text { post-lesion } \\
14 \text { days }\end{array}$ & $\begin{array}{c}\text { G28d } \\
\text { post-lesion } \\
28 \text { days }\end{array}$ \\
\hline $\begin{array}{c}\text { la } \\
n=6\end{array}$ & $\begin{array}{c}\text { sh } \\
n=3\end{array}$ & $\begin{array}{c}\text { ct } \\
n=3\end{array}$ & $\begin{array}{c}\text { la } \\
n=6\end{array}$ & $\begin{array}{c}\text { sh } \\
n=3\end{array}$ & $\begin{array}{c}\text { ct } \\
n=3\end{array}$ & $\begin{array}{c}\text { la } \\
n=6\end{array}$ & $\begin{array}{c}\text { sh } \\
n=3\end{array}$ & $\begin{array}{c}\mathrm{ct} \\
\mathrm{n}=3\end{array}$ & $\mathrm{n}=5$ & $\mathrm{n}=8$ & $\mathrm{n}=7$ & $\mathrm{n}=7$ \\
\hline
\end{tabular}

$0^{+}$day $=$immediately after partial laceration of right lateral gastrocnemius (RLG).

la = laceration of RLG, sh = sham, skin incision and dissected RLG, ct = control, no lesion.

\section{Laceration protocol}

The laceration protocol implemented in the present work, corresponding to a modified version of a previous one elaborated by Menetrey et al. ${ }^{9}$ for the rat soleus muscle, was applied to animals from sub-group ci in GI, GII e GIII as well as to those in G04d, G14d e G281.

Initially, an intraperitoneal administration of ketamine $(50 \mathrm{mg} / \mathrm{kg})$ associated with xylasine $(5 \mathrm{mg} / \mathrm{kg})$ was followed by shaving both legs and a lateral skin incision, approximately 0.5 $\mathrm{cm}$ long, at the right leg. The femoral biceps was sectioned, with a curved $10 \mathrm{~cm}$ Halstead Mosquito ${ }^{\circledR}$ forceps, the right LG exposed, entirely dissected and its lateral dimension measured. A vessel loop with blunt needle (Surg-I-Loop Plus Vascular loop; Scalan, Saint Paul, MN, EUA) transfixed the LG venter at a midway point, its half side was pulled out, gripping the vessel loop, and entirely sectioned with a curved $12 \mathrm{~cm}$ Noyes Iris scissor (Figure 1). The LG intact half side was measured; hemostasis revision done and skin sutured with nylon monofilament 4-0 seam.
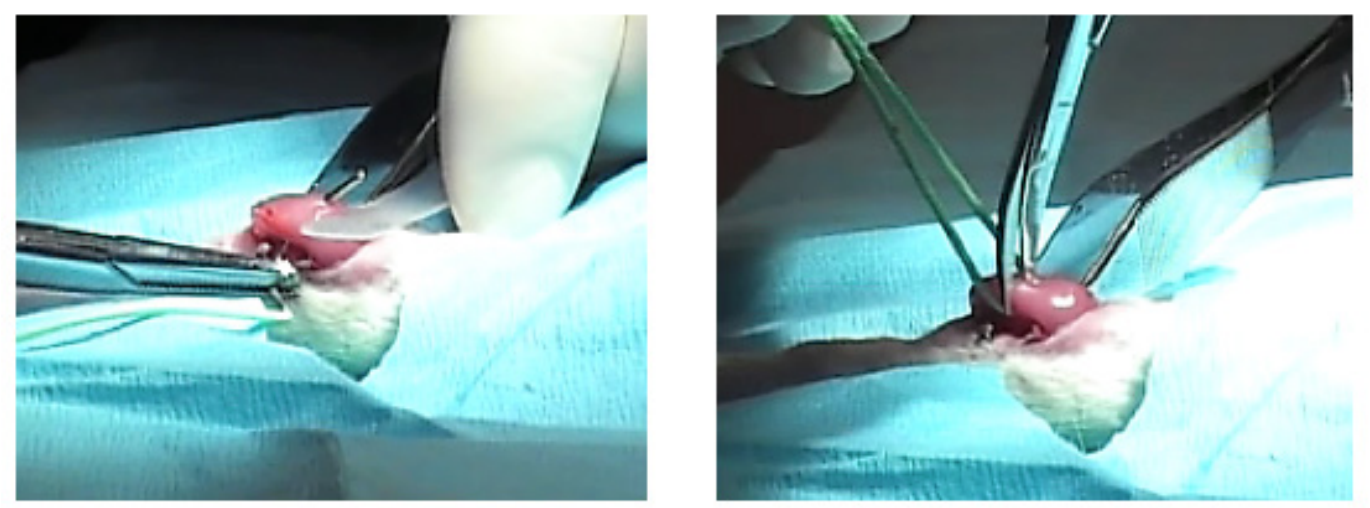

FIGURE 1 - Main steps in the surgical procedure for the laceration of the rat right lateral gastrocnemius (LG) muscle. (Left) LG muscle transfixing with a blunt needle of a vessel loop. (Right) Gripping the vessel loop to pull out the right LG half side and its entire sectioning using the curved $12 \mathrm{~cm}$ Noyes Iris scissor.

\section{UBM image acquisition and inspection}

UBM images were acquired with the $\mathrm{Vevo}^{\circledR} 770$ (Visualsonics; Toronto, ON, Canada) equipment working with a center frequency of $40 \mathrm{MHz}$ and using the probe RMV704 (Visualsonics; Toronto, ON, Canada). This equipment generates ultrasound images with lateral and axial resolutions of 80 and 40 $\mu \mathrm{m}$, respectively, at a frame rate of 34 frames/second.

The animals la1-la6 and sh1-sh3 in GI sub-group were inspected immediately after the surgical intervention and therefore under the intraperitoneal anesthesia. The remained animals were inspected under isoflurane anesthesia at $1.5 \%$ in $1.5 \mathrm{~L} / \mathrm{min}$ oxygen, using a laboratory animal compact table $\mathrm{Vevo}^{\circledR}$ anesthesia system (Visualsonics; Toronto, ON, Canada).

The animals were placed over an animal platform (Visualsonics; Toronto, ON, Canada) in prone position, with ankle angle of and $150^{\circ}$ (Figure 2) imposed by the use of an external fixation. 


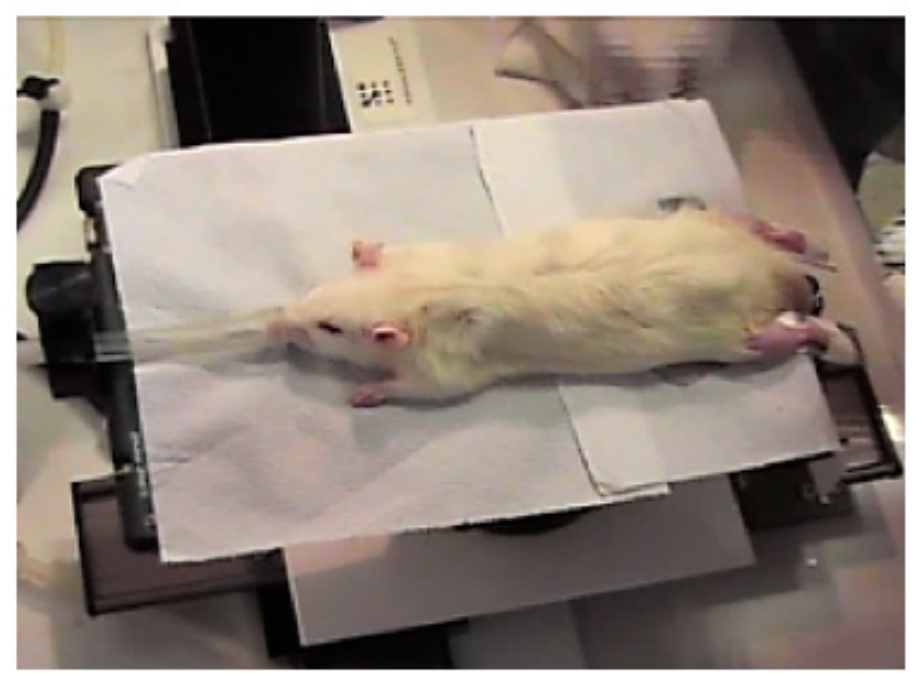

FIGURE 2 - Animal under inhalatory anesthesia, prior to UBM image acquisition, in prone position, over an animal platform, with ankle angles of and $150^{\circ}$ imposed by the use of an external fixation.

The UBM images were acquired, by the same machine operator, for both legs and on a longitudinal plane, related to force generation axis of the gastrocnemius muscle. Once the muscle was visualized in the image, then a 100 frames video clip was saved and thereafter 3 frames randomly selected for analysis by an experienced radiologist. The method of analysis, even for injured or healthy muscle, based on image characteristics such as the image general quality, the contrast and the echogenicity. Regarding the lacerated muscle, the boarders of the lesion were defined accounting for the retracted and hyperechoic muscle fibers visualized in the images.

\section{Contractility tests of an isolated LG muscle}

The LG muscles of all animals in sub-group $\mathrm{G}_{\mathrm{cmi}}$ were submitted to contractility test, ex vivo, to measure the twitch under an external electrical stimulation. During the test, the muscle was immersed in a reservoir filled with Ringer-Krebs solution at the temperature of $35^{\circ} \mathrm{C}$ and composed of (in $\mathrm{mM}$ ) NaCl 118.1; $\mathrm{KCl}$ 3.4; $\mathrm{MgCl}_{2} 1 ; \mathrm{CaCl}_{2} 2.5 ; \mathrm{NaHCO}_{3} 25 ; \mathrm{NaHPO}_{4}$ 1.2; dextrose 11 and oxygenated with carbogen mixture $\left(95 \% \mathrm{O}_{2}\right.$ plus $5 \% \mathrm{CO}_{2}$. The $\mathrm{pH}$ was maintained at 7.4 while equilibrated with carbogen mixture.

The muscle was mounted in vertical reservoir, with one extremity attached to a force transducer (TRI201 ${ }^{\circledR}$; Letica Scientific Instruments, Barcelona, Spain) and the other to a fixed clamp. Two platinum circular electrodes (12 $\mathrm{mm}$ in diameter and $28 \mathrm{~mm}$ apart) surrounded the muscle and were used for electrical stimulation.
These electrodes were fed by a general purpose stimulator ( $\mathrm{S} 88^{\circledR}$; Grass Technologies, Warwick, RI, USA).

The force transducer output was connected to a 4 channels quad bridge amplifier (ML224 ${ }^{\circledR}$ Quad Bridge Amp; ADInstruments Ltd, Dunedin, New Zealand) and the amplified signal transmitted to a signal digital acquisition unity $\left(\right.$ PowerLab $^{\circledR}$ 8/35; ADInstruments Ltd, Dunedin, New Zealand), with 8 independent acquisition channels, each with a 16 bits analog to digital converter able to operate at maximum sample rates of 100 $\mathrm{kHz}$, when all 4 channels are used at the same time. The digitized signals were transferred, by USB interface, to a microcomputer for signal processing, with a dedicated software $\left(\right.$ LabChart ${ }^{\circledR}$ version 7.0; ADInstruments Ltd, Dunedin, New Zealand).

After resting in the solution during 15 minutes, the muscle was excited following a calibrating protocol, to verify linearity of the muscle mechanical response to different electrical potentials between the two electrodes. This protocol considered electrical stimulation pulses with $0.2 \mathrm{~ms}$ in duration and repetition frequency of $0.2 \mathrm{~Hz}$ and amplitudes varying from 10 to $50 \mathrm{~V}$ in steps of $10 \mathrm{~V}$. Once confirmed the linear response of the muscle, then $50 \mathrm{~V}$ was maintained between the electrodes and the mechanical tension in the cable attaching the muscle to the force transducer adjusted to maximize the twitch amplitude. Achieved the maximization of the twitch amplitude then the baseline offset of the signal representing the muscle force was compensated, by software, in order to bring the baseline to zero.

Finished the calibration protocol, then the muscle was kept excited with electric pulses with duration of $0.2 \mathrm{~ms}, 50 \mathrm{~V}$ between the electrodes, and during 15-30 minutes. Thereafter, the stimulation followed the protocol (Figure 3 ) and the signals representing the force generated by the muscle were captured and save during the entire period of the protocol.

The muscle (injured or not) mechanical response was analyzed in terms of the twitch amplitudes $P t_{\text {pre }}$ e $P t_{\text {post }}$ related to pre and post $T_{F i}$, respectively, with $T_{F i}$ representing the intervals $T_{F 1}$ to $T_{F 8}$, in which the stimulation frequency varied from 0.2 to $100 \mathrm{~Hz}$, in accordance to the scheme in Figure 3. The values of $P t_{\text {pre }}$ and $P t_{\text {post }}$ refer to the mean of 3 to 4 amplitudes of consecutive twitches. 


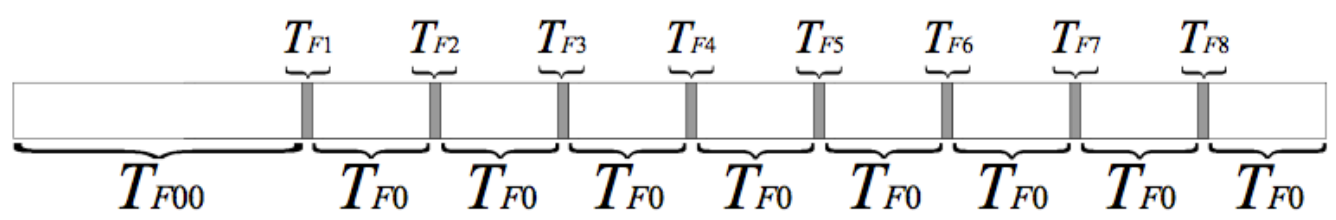

FIGURE 3 - Sequence of the stimulation electrical pulses, including their duration and frequency: $T_{\mathrm{F} 00}(15-20 \mathrm{~min}, 0.2 \mathrm{~Hz}) ; T_{\mathrm{F} 0}(5 \mathrm{~min}$, $0.2 \mathrm{~Hz}) ; T_{\mathrm{F} 1}(5 \mathrm{~s}, 0.5 \mathrm{~Hz}) ; T_{\mathrm{F} 2}(5 \mathrm{~s}, 1.0 \mathrm{~Hz}) ; T_{\mathrm{F} 3}(5 \mathrm{~s}, 5.0 \mathrm{~Hz}) ; T_{\mathrm{F} 4}(5 \mathrm{~s}, 10.0 \mathrm{~Hz}) ; T_{\mathrm{F} 5}(5 \mathrm{~s}, 30.0 \mathrm{~Hz}) ; T_{\mathrm{F} 6}(5 \mathrm{~s}, 50.0 \mathrm{~Hz}) ; T_{\mathrm{F} 7}(5 \mathrm{~s}, 70.0 \mathrm{~Hz}) ;$ $T_{\mathrm{F} 8}(5 \mathrm{~s}, 100.0 \mathrm{~Hz})$.

\section{Death induction}

The animals were induced to death under anesthesia and $\mathrm{CO}_{2}$ inhalation.

\section{Histological analysis}

Dissected and excised right and left LG muscles from animals in group $\mathrm{G}_{\mathrm{bmu}}$ were processed, following standard staining procedures, to provide histological slides containing either transversely or longitudinally sectioned $(5 \mu \mathrm{m})$ muscle samples stained with hematoxylin and eosin (HE) and with picro-sirius. HE staining was used for qualitative evaluation of topography, inflammatory infiltrate, degenerated or necrotic fibers or granulation tissue. On the other hand, picro-sirius staining was used for qualitative evaluation of topography and the presence of collagen.

\section{Statistical analysis}

Comparison between the contractility test results of animals in group $\mathrm{G}_{\mathrm{cmi}}$, was based on statistical analysis using the software BioEstat 5.3, considering $\mathrm{p}<0.05$ for a statistical significant difference between the results.

The Shapiro-Wilk test was initially applied to verify if the data of same sample had a normal distribution. The samples with normal distribution were analyzed testing their variance with the parametric one-way ANOVA test (if the sample variances were not different, $\mathrm{p}<0.05$ ). When the ANOVA detected samples statistically different then the sample means were tested two by two using the Tukey post-hoc test, to identify the pair of samples with different means. For those samples without a normal distribution, or even when their variances were different $(p<0.05)$, then the non-parametric Kruskal-Wallis test, followed by the post-hoc Student-Newman-Keuls test for multiple comparisons, was used to determine significant differences among the sample means.

\section{Results}

\section{UBM images}

Typical UBM images, in sagittal plane, of the right LG muscle are presented for rats in $\mathrm{G}_{\mathrm{bmu}}$-ct (Figure 4), $\mathrm{G}_{\mathrm{bmu}}$-sh (Figure 5) and $\mathrm{G}_{\mathrm{bmu}}$-la (Figure 6) sub-groups. Figures 5 and 6 display images acquired immediately, 14 and 28 days after surgery.

In general, the image of animals in $\mathrm{G}_{\mathrm{bmu}}$-ct subgroup (Figure 4), presented preserved echo texture and muscle architecture, well defined fascicles, parallel and rectilinear equidistant hyperechoic lines (perimysium)

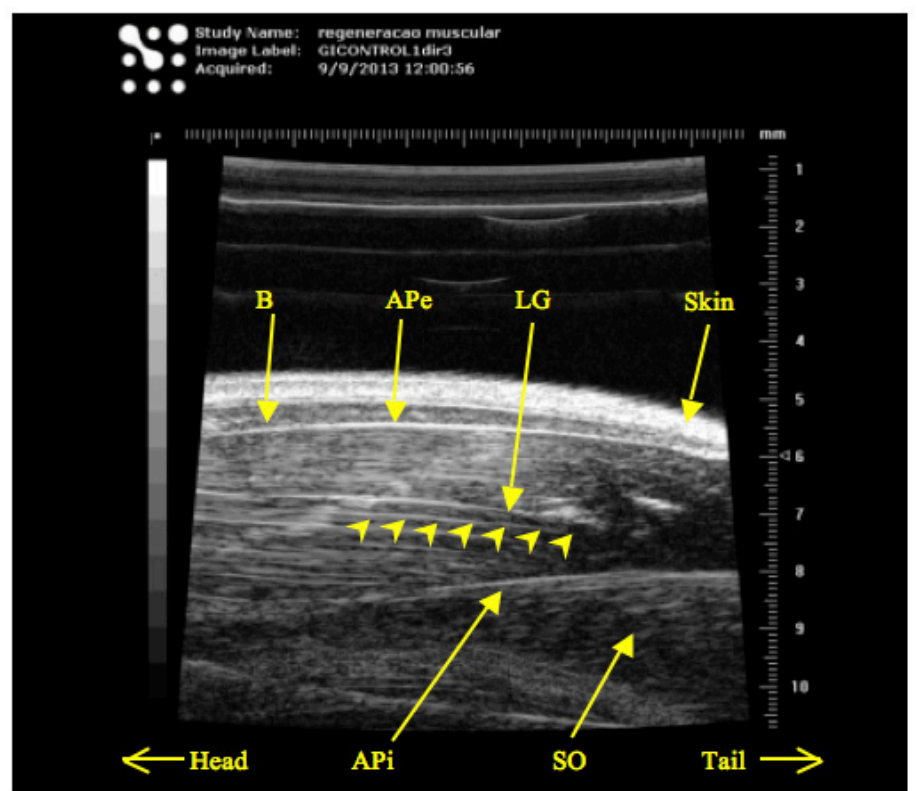

FIGURE 4 - Typical $40 \mathrm{MHz}$ UBM image, in sagittal plane, of the rat right lateral gastrocnemius (LG) from the control sub-group $\left(\mathrm{G}_{\mathrm{bmu}}{ }^{-\mathrm{ct}}\right)$. Anatomic details clearly observed: skin, biceps femoral $(\mathrm{BF}), \mathrm{LG}$, soleus (SO), external aponeuroses (APe), between LG e $\mathrm{BF}$, and internal (APi), between LG e SO. The hyperechoic traces relate to the perimysium (arrowheads). Scale: $0.1 \mathrm{~mm} /$ division. 
The UBM images of LG muscle for the animals in $\mathrm{G}_{\mathrm{bmu}}$-sh sub-group present a heterogeneous echo texture for the biceps femoral (Figure 5A), if compared to $\mathrm{G}_{\mathrm{bmu}}$-ct sub-group, the lack of clear boundary between biceps femoral and LG muscles (Figure 5A) and the LG echo texture is found preserved 14 (Figure 5B) and 28 (Figure $5 \mathrm{C}$ ) days after surgery, with identification of the fibrillar arrangement.
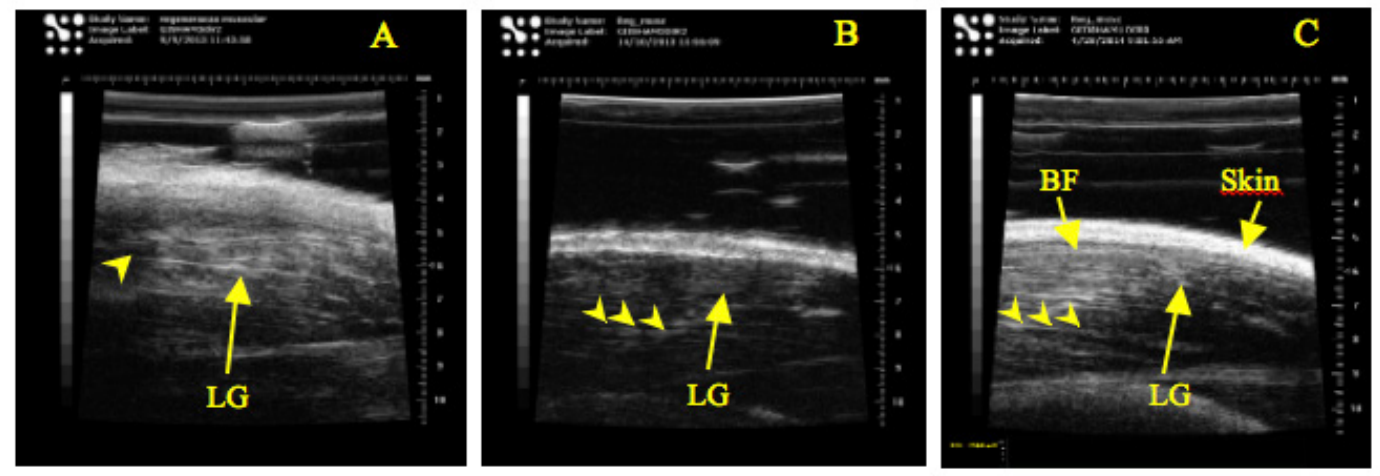

FIGURE 5 - 40 MHz UBM image, sagittal plane, of the rat right lateral gastrocnemius (LG) from the false operated sub-group (sham, $\mathrm{G}_{\mathrm{bmu}}$-sh). Images acquired immediately - GI (A), 14 days - GII (B) and 28 days - GIII (C), after surgery. Biceps femoral (BF) echo texture in $(\mathbf{C})$ preserved, while in (A) is heterogeneous (arrowhead), with difficulty to delineate the interface with LG. Echo texture preserved, with fibrillar arrangement in LG (arrowheads in B and C). Scale: $0.1 \mathrm{~mm} /$ division.

Regarding the animals in lacerated sub-group $\left(\mathrm{G}_{\mathrm{bmu}}-\mathrm{la}\right)$, decreased in comparison to the same region in the image acquired it is observed (Figure 6) the partial rupture of the LG muscle, immediately after the laceration of the LG muscle. Also, the corresponding to the area where the fascicles are not continuous repaired muscle fascicles started to show up. Twenty-eight days and a liquid fills the region between the lacerated extremities after surgery, the muscle fascicles present a complete repair, (hypoechoic area). Fourteen days after surgery, the lesion area is oriented and forming the pennation angle as in the health muscle.
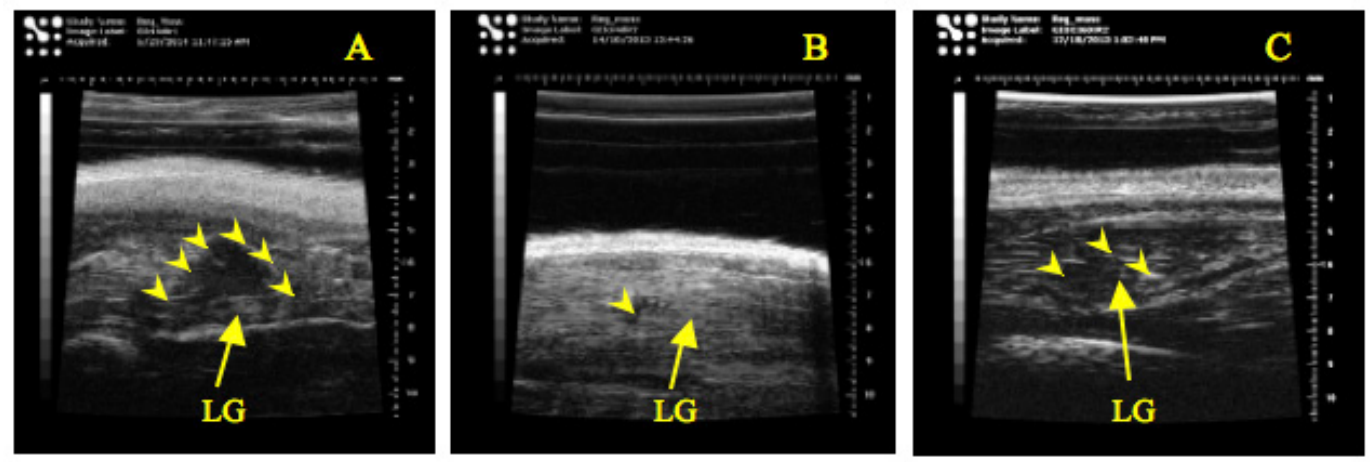

FIGURE 6 - 40 MHz UBM image, sagittal plane, of the rat right lateral gastrocnemius (LG) from the lacerated sub-group, $\mathrm{G}_{\text {bmu }}$-la. Images acquired immediately - GI (A), 14 days - GII (B) and 28 days - GIII (C) after surgery. (A) Partial rupture of LG in the area, hypoechoic and limited by the arrowheads, filled with liquid and fascicles without continuity. (B) Injured area (arrowhead) decreased in comparison to the same region as in (A) and the presence of repaired muscular fascicles. (C) Muscle fascicles repaired, oriented and forming the pennation angle as in the health muscle (arrowhead). Scale: $0.1 \mathrm{~mm} /$ division.

\section{Contractility test}

The amplitudes $T D_{\text {pre }}$ and $T D_{\text {post }}$ of the mechanical tension corresponding to twitches $P T_{\text {pre }}$ and $P T_{\text {post }}$, respectively, and generated by the gastrocnemius muscles of animals in control sub-group (Gct) were compared with those related to the animals from sub-groups G04d, G14d and G28d, submitted to laceration of the right $\mathrm{LG}$. The mechanical tension amplitudes were determined from the amplitudes of $P T_{\text {pre }}$ and $P T_{\text {post }}$ divided by the crosssectional area of the corresponding muscle and multiplied by the gravity acceleration.

The mean $( \pm \mathrm{std})$ of the gastrocnemius muscles of all animals in $\mathrm{G}_{\mathrm{cmi}}$ group involved in contractility tests was $0.62( \pm 0.15)$ and $0.62( \pm 0.14)$ for left and right muscles, respectively. There was no statistical difference $(\mathrm{p}<0.05)$ between the mean cross-sectional area of both muscle sides. 
The results (Table 2) are presented in terms of comparison between the mechanical tension amplitudes generated by the LG muscle and due to an external electrical stimulation. In general, there was no statistical difference $(\mathrm{p}<0.05)$ between the mechanical tension amplitudes, regardless of the muscle sides and the mechanical tension amplitudes measured before and after the intervals $\mathrm{T}_{\mathrm{F} 1}-\mathrm{T}_{\mathrm{F} 8}$ intervals. Statistical significant differences only occurred when $T D_{\text {pre }}$ and $T D_{\text {post }}$ for the right LG, between muscles of animals in control and injured groups, with $T D_{\text {pre }}$ referring to stimulating frequency of $50 \mathrm{~Hz}$ and with 4 days after surgery, as well as for $T D_{\text {post }}$ referring to stimulating frequencies of 70 and $100 \mathrm{~Hz}$ and with four or 14 days after laceration.

TABLE 2 - Results of statistical tests comparing the mechanical tension between non-injured and injured gastrocnemius muscles.

\begin{tabular}{|c|c|c|c|c|}
\hline & Get & G04d & G14d & G28d \\
\hline Gct & & $\begin{array}{c}\mathrm{p}<0.05: \\
T D_{\text {pre }}, 50 \mathrm{~Hz} \\
T D_{\text {post }}, 70 \text { and } 100 \mathrm{~Hz}\end{array}$ & ns & ns \\
\hline G04d & $\begin{array}{c}\mathrm{p}<0.05: \\
T D_{p r e}, 50 \mathrm{~Hz} \\
T D_{p o s t}, 70 \text { and } 100 \mathrm{~Hz}\end{array}$ & & $\begin{array}{c}\mathrm{p}<0.05: \\
T D_{\text {post }}, 70 \text { and } 100 \mathrm{~Hz}\end{array}$ & ns \\
\hline G14d & ns & $\begin{array}{c}\mathrm{p}<0.05: \\
T D_{\text {post }}, 70 \text { and } 100 \mathrm{~Hz}\end{array}$ & & ns \\
\hline G28d & ns & ns & ns & \\
\hline
\end{tabular}

$T D_{\text {pre }}=P T_{\text {pre }} \cdot{ }^{\circ} / \mathrm{S}, T D_{\text {post }}=P T_{\text {post }} \cdot{ }^{\circ} / \mathrm{S}, g=$ gravity acceleration, $S=$ transverse sectional area, $P T_{\text {pre }}$ and $P T_{\text {post }}=$ twitch amplitudes pre and post change in stimulating frequency, Gct, G04d, G14d, G28d = contractility test of non-injured muscle and 4, 14 and 28 days post-laceration, respectively.

\section{Histopathological analysis}

The skeletal LG muscle tissue of animals in control and sham sub-groups (4, 14 and 28 days post-laceration) did not present any histological changes, with tissue structure formed by elongated myocyte bundles, when longitudinally sectioned, with peripheral nucleus of even size and form. The histological findings agree with corresponding UBM images (Figures 4 and 5C), in which the muscle fascicles form hyperechoic parallel and equally spaced lines (perimysium, indicated by arrowheads).
The right LG muscle of animals in sub-group G04d (four days after lesion) presented an area of muscular discontinuity, filled with inflammatory infiltrate (Figure 7A) interposed with degenerated or necrotic fibers (Figure 7B). It is also noticed tissue regions containing granulation tissue formed with basophilic fibroblast-like and stellate cells dispersed among an edematous and vascularized tissue (Figure 7C). The UBM images (Figure 6A) of the right LG muscles of animals from this group also exhibited partial rupture of LG muscle, visualized by fiber disconnections surrounding an anechoic region filled with liquid (arrowheads).
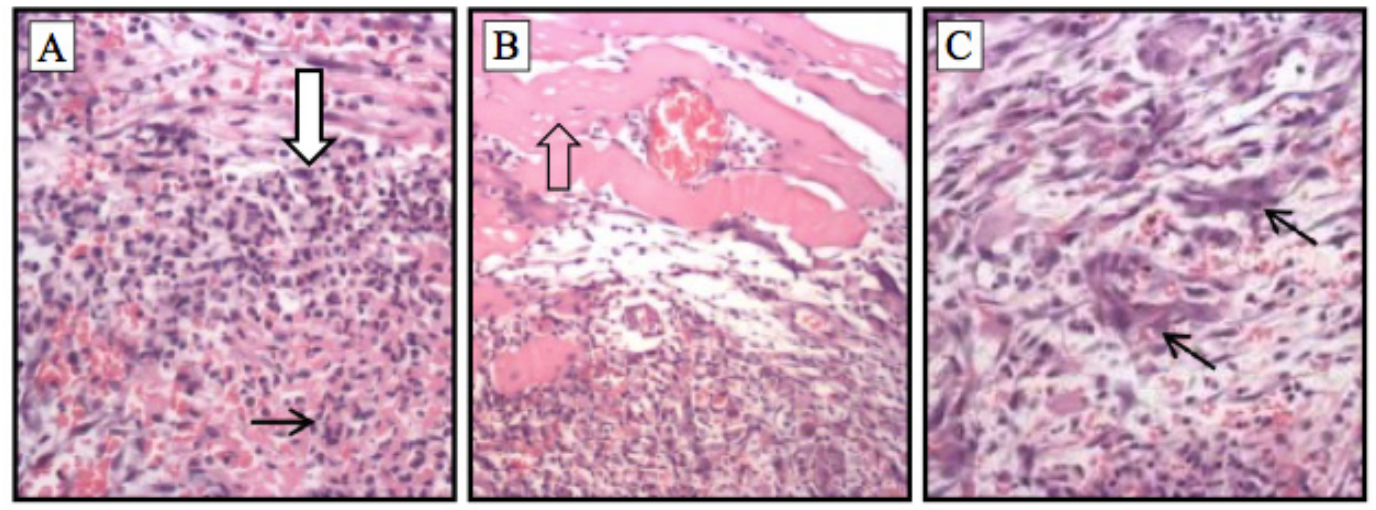

FIGURE 7 - Photomicrography of the rat right gastrocnemius muscle, lacerated sub-group with four days after surgery, and stained with hematoxylin and eosin. (A) Lesion site with inflammatory infiltrate (black arrow), neutrophils among cellular debris (arrow filled in white) and mononucleated cells. (B) Inflammatory infiltrate aside muscular fibers in degeneration (vacuolated aspect, no transverse striae and intensively eosinophilic - unfilled arrow). (C) Presence of star-like basophilic fibroblasts (arrows). Original enlargement: x200 in (A) and (C), x100 in (B). 
Fourteen days after surgery, the injured site exhibited an intense mononucleated type inflammatory infiltrate, among granulation tissue (interstitial edema, abundant and congested capillaries) containing myocyte fragments (Figure 8A). The presence of basophilic cells over the area of discontinuous muscular architecture was noticed with augmented nucleuscytoplasm ratio, evident nucleolus, that correspond to immature cells (slightly differentiated, myoblasts) and loose chromatin (Figure 8A). Besides the loosened areas, it was also noticed that sites with more cells were present in the discontinuous myofibers, which were infiltrated by mononucleated type inflammatory cells, basophilic fibroblast-like or stellate cells. In some cases, the myoblasts presented a more extended format, installing aside the myocytes (Figure 8B). In addition, the vacuoles inside the degenerating myocytes seemed to coalesce, forming intracellular channels (Figure 8B) that are populated with macrophages and basophilic cells. The re-establishment of myocyte rows seemed to be formed from myoblasts fused with degenerating myocytes, forming multinucleated structures in the corresponding region (Figure 8C). The picro-sirius staining reveals collagen, in red, deposited in the repairing area of the lesion site (Figure 9A), where smaller myocytes (young myocytes) are evidenced, yet disorganized in relation to the muscular-tissue architecture. Rare adipose cells are also found among the deposited collagen (Figure 9A). Myocytes with several nucleus on its extremity are also observed (Figure 9B), emphasizing a repairing process that dependent on preexisting myocytes fused with myoblasts. Part of these histologic findings are in correspondence with the UBM image (Figure 6 B), depicting a minor amount of ruptured fibers, with smaller and better defined hypoechoic region when compared with the image acquired four days post injury.
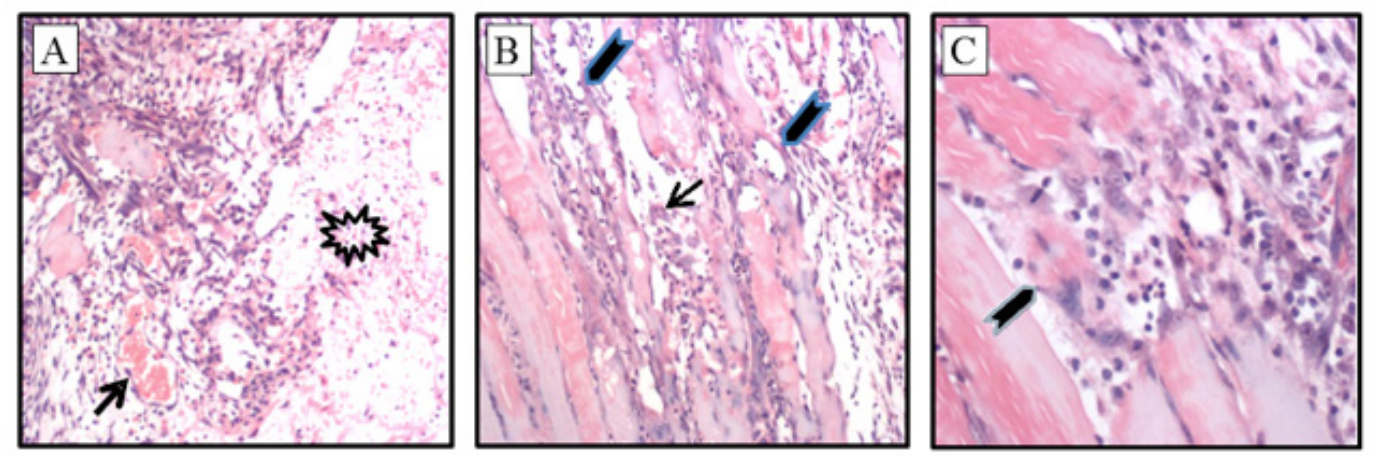

FIGURE 8 - Photomicrography of the rat right gastrocnemius muscle, lacerated sub-group with 14 days post-surgery, and stained with hematoxylin and eosin. (A) Loosened connective tissue with several capillaries (dark arrow), granulomatous tissue and mononucleated cells (surrounded by blue spiculated trace). (B) Vacuoles forming in the boundaries of degenerating myocytes (large dark arrows) kept apart by basophilic cells (small dark arrow). (C) Presence of elongated cells, with basophilic cytoplasm, aligned in the lesion site, installed sideway of the degenerating muscle (dark large arrow) and filling the vacuoles. Original enlargement: x200 in (A), (B) and (C).
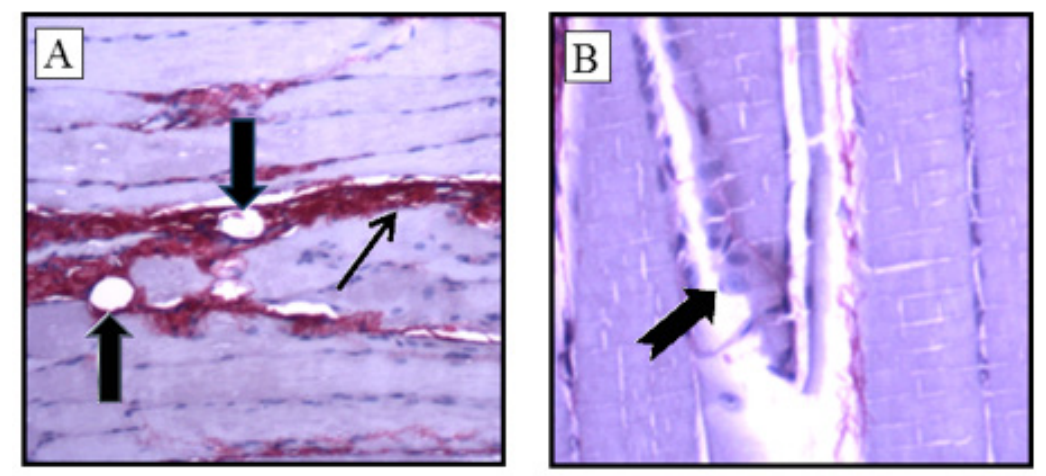

FIGURE 9 - Photomicrography of the rat right gastrocnemius muscle, lacerated sub-group with 14 days post-surgery, and stained with picro-sirius. (A) Increased collagen deposition between myocytes (dark arrow) and adipocytes (larger dark arrow). (B) Myocyte extremity with multinucleation, indicating repair. Original enlargement: x100 in (A) and x200 in (B). 
Twenty-eight days post-trauma, the right LG muscles from the animals in injured sub-group exhibited, through histological analysis, the re-establishment of the normal architecture, with all myocytes grouped parallel with respect to each other and having fusiform nucleus and dense chromatin (Figures 10A and B). Exceptionally, only one fiber, among all others in the muscular fascicle present in Figure 9A, exhibited peripheral (Figure 10A) or centered (Figure 10B) enlarged nucleus with loose chromatin, representing young myocytes. Muscle repairing is also noticed in corresponding UBM image (Figure 6C), in which the muscle exhibits a minor disorganization of myofibrillar architecture.
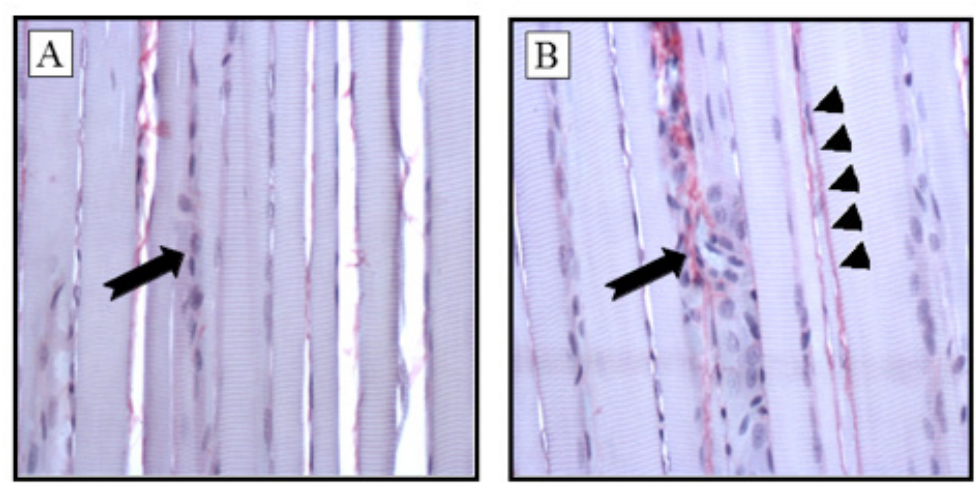

FIGURE 10 - Photomicrography of the rat right gastrocnemius muscle, lacerated sub-group with 28 days after surgery, and stained with picro-sirius. (A) and (B) myocytes settled longitudinally nucleus and some of the cells with more centered and egg-shaped nucleus (large dark arrows), related with young myocytes. Collagen deposition, colored red, around the fibers (arrowhead). Original enlargement: $\mathrm{x} 200$ in $(\mathbf{A})$ and (B).

\section{Discussion}

Models of muscle lesion and repair process are fundamental for new discoveries that may enable therapeutic strategies for treatment of muscular diseases or even damages caused by accidents that cause suffering as well as losses. So far, literature reports related to muscle laceration protocol do not describe, in full detail, neither the lesion models regarding anatomical aspects corresponding to the muscle width in a certain topography, nor how they measured the lesion dimensions. Examples of published reports include the work by Menetrey et $a l .{ }^{9}$ and by Peixinho et al. ${ }^{13}$. The muscle laceration procedures employed in the previous two works were modified, in the present work, to standardize the surgical procedure in order to provide better control of the lesion dimension and location. In this regard, the rat right lateral gastrocnemius was exposed and entirely dissected, its width measured using a scale ruler with millimeter precision, and the half lateral side entirely sectioned. Precautions regarding anesthesia, standard post-surgery technique and the experience acquired with the rat gastrocnemius anatomy, nurtured the safety and controlled characteristics of the experimental work. The implemented lesion protocol achieved some characteristics such as: safety, control, precision and reproducibility.

In addition to the lesion protocol, the present work also contributed to following-up of the muscle repairing process based on the three methods: acquisition of UBM images using an instrumentation adequately scaled for small animals (rat or mouse), the measurement of muscle force induced with external electrical stimulation (ex vivo) and histopathological analysis.

In order to obtain well-defined UBM ultrasound images from the site of the muscle acute lesion, the region of damaged tissue needs to be determined topographically, with precise location. The injured area, characterized as a hypoechoic UBM image (hematoma) between the extremities of the discontinuous myofibers, was confirmed throughout the entire experiments involving the laceration protocol, attesting in this way a reproducible and precise lesion model.

Excellent correspondence between the lesion site and its depiction by the UBM image, as well as the findings along the following-up of the muscle repair, was excellent. Additionally, there is a correspondence between the descriptive analysis of muscle repair, based on the cytomorphological representation obtained from histology, and on the finds from the UBM images acquired form LG right muscle of animals with 14 and 28 days post-surgery.

The analysis of the injured muscle repair/regeneration based on the histological findings revealed that the degenerated myofiber boundaries, as well as its interior, acted as a scaffold to serve the myoblast migration for the fusion of the new myofibrils. Tidbal and Villalta ${ }^{14}$ already mentioned this finding of muscular 
repairing, however without documented demonstration.

Regarding the contractility tests, most of the results related to the force generated by the gastrocnemius muscle, ex vivo, in response to an electrical stimulation did not present statistical significant differences, when comparing health and injured muscles from the right side, between muscles from right and left sides from the same animal and only muscles from the left side. Nevertheless, significant differences occurred in two situations; at stimulating electrical signals with frequencies of 50 and $70 \mathrm{~Hz}$, in which tetanic response existed. In such situations, there was difference $(\mathrm{p}<0.05)$ when comparing the results between the right side muscles of animals in control and injured subgroups with four and 14 days after surgery. The explanation of this finding is still unclear and deserves further investigation, which is beyond the context of the present work.

The work by Peixinho et al. ${ }^{13}$ observed a compensatory hypertrophic response, related to increased force production, of the healthy contralateral limb and this finding may have been caused by an increased load during the muscle repair period of injured member. However, the finding by Peixinho et al. ${ }^{13}$ was not confirmed by the contractility tests performed in the present work, when comparing the twitches between left and injured right LG muscles, with the measurements done at four, 14 and 28 days post-laceration. The reason for this may be the variability in the measurement of twitches and inherent to contractility tests. More reliable measurements may be necessary in order to avoid variability of the measured data to overcome the difference in twitches between healthy and injured muscles.

The results obtained with UBM and histology methods of the rat injured gastrocnemius and at 28 days after surgical intervention agreed with other; the muscle was under an advanced stage of repair. The results of the contractility test, also obtained 28 days post-surgery, provide the same interpretation of those from UBM and histology methods, since the test did not detect functional changes of the muscle when compared with the results from the healthy muscle. Most of the evidences of damage in the injured muscle, detected together from UBM images, histological findings and contractility test, were detected between four and 14 days following the muscle laceration.

Considering the correspondence of the findings obtained with UBM, histology and contractility test methods, then UBM becomes a potential method to be employed in future works related to the following-up of muscle repair, boosted by the advantages of the method, including non-invasiveness and suitability for in vivo applications that allow for longitudinal investigations, as opposed by the other two methods.

\section{Conclusions}

A protocol for laceration of the rat right gastrocnemius was implemented and is ready to be used safely and in controlled and reproducible ways. Additionally, the findings from ultrasound biomicroscopy images, tests of muscle contractility and histology have correspondence in respect to the following up of injured muscle repair.

\section{References}

1. Marini M, Veicsteinas A. The exercised skeletal muscle: a review. Eur J Transl Myol. 2010;20:105-20. doi: 10.4081/bam.2010.3.105.

2. Jarvinen MJ, Lehto MU. The effects of early mobilisation and immobilisation on the healing process following muscle injuries. Sports Med. 1993;15(2):78-89. PMID: 8446826.

3. Jarvinen TA, Jarvinen TL, Kaariainen M, Aarimaa V, Vaittinen S, Kalimo H, Jarvinen M. Muscle injuries: optimising recovery. Best Pract Res Clin Rheumatol. 2007;21:317-31. PMID: 15851777.

4. Ceafalan LC, Popescu BO, Hinescu ME. Cellular players in skeletal muscle regeneration. Biomed Res Int. 2014;2014:957014. doi: 10.1155/2014/957014.

5. Grefte S, Kuijpers-Jagtman AM, Torensma R, Von den Hoff JW. Model for muscle regeneration around fibrotic lesions in recurrent strain injuries. Med Sci Sports Exerc. 2010;42:813-9. doi: 10.1249/ MSS.0b013e3181beeb52.

6. Schoenfeld BJ. The use of nonsteroidal anti-inflammatory drugs for exercise-induced muscle damage: implications for skeletal muscle development. Sports Med. 2012;42(12):1017-28. doi: 10.2165/11635190-000000000-00000.

7. Sicari BM, Dearth CL, Badylak SF. Tissue engineering and regenerative medicine approaches to enhance the functional response to skeletal muscle injury. Anat Rec (Hoboken). 2014;297:51-64. doi: 10.1002/ar.22794.

8. Crisco JJ, Jokl P, Heinen GT, Connell MD, Panjabi MM. A muscle contusion injury model. Biomechanics, physiology, and histology. Am J Sports Med. 1994;22:702-10. PMID: 7810797.

9. Menetrey J, Kasemkijwattana C, Fu FH, Moreland MS, Huard J. Suturing versus immobilization of a muscle laceration. A morphological and functional study in a mouse model. Am J Sports Med. 1999;27:222-9. PMID: 10102105.

10. Markert CD, Merrick MA, Kirby TE, Moreland MS, Huard J. Nonthermal ultrasound and exercise in skeletal muscle regeneration. Arch Phys Med Rehabil. 2005;86:1304-10. PMID: 16003655.

11. Hwang JH, Kim IG, Piao S, Jung AR, Lee JY, Park KD, Lee JY. Combination therapy of human adipose-derived stem cells and basic fibroblast growth factor hydrogel in muscle regeneration. Biomaterials. 2013;34:6037-45. doi: 10.1016/j. biomaterials.2013.04.049.

12. Souza J, Gottfried C. Muscle injury: review of experimental models. J Electromyogr Kinesiol. 2013;23:1253-60. doi: 10.1016/j. jelekin.2013.07.009

13. Peixinho CC, Ribeiro MB, Resende CM, Werneck-de-Castro JP, de Oliveira LF, Machado JC. Ultrasound biomicroscopy for biomechanical characterization of healthy and injured triceps surae of rats. J Exp Biol. 2011;214:3880-6. doi: 10.1242/jeb.059808.

14. Tidball JG, Villalta SA. Regulatory interactions between muscle and the immune system during muscle regeneration. Am J Physiol Regul Integr Comp Physiol. 2010;298(5):R1173-87. doi: 10.1152/ ajpregu.00735.2009. 


\section{Acknowledgements}

The technical support of Alyson do Rosario Junior and José Nazioberto Duda de Farias for preparing the histological slides of Dr. Luiz Brasílio, the analysis of UBM images, and of Cinthia Cruz, for the handling with animals and experiments.

\section{Correspondence:}

Prof. Dr. João Carlos Machado

Universidade Federal do Rio de Janeiro

Avenida Horácio Macedo, 2030

Centro deTecnologia,Bloco H/sala 327

21941-914 Rio de Janeiro - RJ Brasil

Tel./Fax: (55 21)3839-8578/3889-8591

jcm@peb.ufrj.br

Received: Sep 10, 2014

Review: Nov 12, 2014

Accepted: Dec 15, 2014

Conflict of interest: none

Financial sources: National Council for Scientific and Technological Development $(\mathrm{CNPq})$, Coordination of Improvement for Higher Academic Staff (CAPES) and Carlos Chagas Filho Foundation for Research Support of the State of Rio de Janeiro (FAPERJ).

${ }^{1}$ Research performed at Experimental Surgery Center, School of Medicine, Department of Basic and Clinical Pharmacology and the National Center of Bioimaging, Federal University of Rio de Janeiro (UFRJ), Brazil. Part of Master degree thesis, Postgraduate Program in Surgical Sciences, UFRJ. Tutor: João Carlos Machado. 